\title{
PERFORMANCE COMPARISON OF DUAL-BRANCH AND TRIPLE-BRANCH SELECTION DIVERSITY RECEIVER BASED ON DESIRED SIGNAL ALGORITHM OVER CORRELATED WEIBULL FADING CHANNELS
}

UDC (621.391.812:621.317.3)

\author{
Aleksandra Golubović ${ }^{1}$, Nikola Sekulović ${ }^{2}$, \\ Mihajlo Stefanović ${ }^{1}$, Dejan Milić ${ }^{1}$ \\ ${ }^{1}$ University of Niš, Faculty of Electronic Engineering, Niš, Republic of Serbia \\ ${ }^{2}$ College of Applied Technical Sciences, Niš, Republic of Serbia
}

\begin{abstract}
As an important performance measure, in this paper the outage probability of dual-and triple-branch selection combining (SC) diversity system is compared. The analyzed system works over correlated Weibull fading channels in the presence of cochannel interference (CCI) where the output signal choice is done using desired signal algorithm. Results are graphically presented showing the influence of number of diversity branches, correlation coefficient and ratio of average powers of desired and interference signal.
\end{abstract}

Key words: Cochannel interference, correlated Weibull fading channels, desired signal algorithm, selection diversity.

\section{INTRODUCTION}

Transmitted signal in wireless communication is corrupted by fading and cochannel interference (CCI) therefore the wireless systems can benefit greatly by channel estimation. Since channel estimation cannot be done perfectly, different fading models are used in order to predict as accurately as possible the signal at the receiving point. The most frequently used statistical models for describing the fading envelope of the received signal are Rayleigh, Rician, Nakagami and Weibull. This paper focuses on Weibull distribution since it is simple and flexible yet not exploited as much as the other models. It represents an excellent fit to experimental fading channel measurements for indoor [1], [2] and outdoor [3]-[5] environments.

Received November 16, 2016

Corresponding author: Aleksandra Golubović

University of Niš, Faculty of Electronic Engineering, Aleksandra Medvedeva 14, 18000 Niš, Republic of Serbia

E-mail: aleksandra321@gmail 
Diversity techniques provide inexpensive way to improve performance of wireless communication. The concept of diversity techniques is simultaneous reception of the same radio signal over two or more paths in order to increase the overall signal-to-noise ratio (SNR) [6]. The diversity paths can be separated by space, frequency or time and in all cases some redundancy in time, frequency and/or spatial domain is required [7]. Compared with other diversity techniques, space diversity is most commonly used technique since it is power- and bandwidth-efficient [8]. If the best of the received signals is selected or if they are properly combined, the outage time can be substantially reduced [9]. Depending on the communication system complexity restrictions and the amount of channel state information (CSI) available at the receiver, there are several principal types of combining techniques within space diversity. Combining techniques like maximal ratio combining (MRC) and equal-gain combining (EGC) require some amount of the channel state information of received signal and separate receiver chain for each branch of the diversity system that results in system complexity increase. On the other hand, selection combining (SC) receiver processes only one of the diversity branches at the time and it is much simpler and cheaper for practical realization [6].

In interference-limited environment, where the level of CCI is sufficiently high compared to noise, one of the following combining algorithms is used: the desired signal algorithm, the signal-to-interference ratio (SIR) algorithm and the total signal algorithm [10]. In this paper the algorithm based on desired signal is analyzed. $L$-branch EGC and MRC receivers operating over non identical Weibull fading channels have been considered in [11]. Performance analysis of digital communications receivers over Weibull fading channels that employ desired signal algorithm was investigated in [12] for dual-branch and in [13] for triple-branch diversity receiver.

SC diversity in interference-limited environment under Weibull fading scenario (desired signal as well as CCI experience Weibull fading) was considered in [11], [14]-[17]. In [14], assuming that diversity branches are uncorrelated, analytical expression for the outage probability was derived. Independent fading assumes antenna elements to be placed sufficiently far apart. However, in practice due to insufficient spacing between antennas when diversity is applied on small terminals, correlation between diversity branches exists. Study investigating performance of dual-branch, triple-branch and $L$-branch SC diversity operating over correlated Weibull fading channels was reported in [15], [16] and [17], respectively.

This paper presents the comparison of outage probability for dual-branch and triple-branch SC diversity system applying desired signal algorithm. Expressions for probability density function (PDF) for dual- and triple-branch at the output of selection combining receiver are presented and applied to analyze and compare the performance of those two systems. In this paper outage probability was used as performance measure indicator. Numerical results obtained for two previously mentioned systems are graphically presented for different correlation coefficient, fading severity and average SIR at the input, showing the system performance change when the number of diversity branches increases from two to three. 


\section{SYSTEM AND CHANNEL MODEL}

We consider a SC diversity system with two and three branches in interference-limited Weibull fading environment. In small size terminals, where diversity systems are applied, it is not possible to separate the branches completely. As a result, there is degradation of diversity gain.

In such case, desired signal envelopes $\left(x_{1}, x_{2}\right)$ and CCI envelopes $\left(y_{1}, y_{2}\right)$ experience correlative Weibull fading with joint (PDF) for dual-branch system [19, eq. (11)]

$$
\begin{aligned}
& f_{x_{1} x_{2}}\left(x_{1}, x_{2}\right)=\frac{\beta_{1} \beta_{2}}{(1-\rho)} I_{0}\left(\frac{2 \sqrt{\rho} x_{1}^{\beta_{1} / 2} x_{2}^{\beta_{2} / 2}}{(1-\rho) \sqrt{\Omega_{\mathrm{d}_{1}} \Omega_{\mathrm{d}_{2}}}}\right) \frac{x_{1}^{\beta_{1}-1} x_{2}^{\beta_{2}-1}}{\Omega_{\mathrm{d}_{1}} \Omega_{\mathrm{d}_{2}}} \exp \left[-\frac{1}{1-\rho}\left(\frac{x_{1}^{\beta_{1}}}{\Omega_{\mathrm{d}_{1}}}+\frac{x_{2}^{\beta_{2}}}{\Omega_{\mathrm{d}_{2}}}\right)\right], \\
& f_{y_{1} y_{2}}\left(y_{1}, y_{2}\right)=\frac{\beta_{1} \beta_{2}}{(1-\rho)} I_{0}\left(\frac{2 \sqrt{\rho} y_{1}^{\beta_{1} / 2} y_{2}^{\beta_{2} / 2}}{(1-\rho) \sqrt{\Omega_{\mathrm{c}_{1}} \Omega_{\mathrm{c}_{2}}}}\right) \frac{y_{1}^{\beta_{1}-1} y_{2}^{\beta_{2}-1}}{\Omega_{\mathrm{c}_{1}} \Omega_{\mathrm{c}_{2}}} \exp \left[-\frac{1}{1-\rho}\left(\frac{y_{1}^{\beta_{1}}}{\Omega_{\mathrm{c}_{1}}}+\frac{y_{2}^{\beta_{2}}}{\Omega_{\mathrm{c}_{2}}}\right)\right],
\end{aligned}
$$

and for triple-branch system [19, eq. (23) for $L=3$ ]

$$
\begin{aligned}
& f_{x_{1} x_{2} x_{3}}\left(x_{1}, x_{2}, x_{3}\right)=\frac{\beta_{1} \beta_{2} \beta_{3}}{\Omega_{d_{1}} \Omega_{d_{2}} \Omega_{d_{3}}(1-\rho)^{2}} \exp \left\{-\frac{1}{1-\rho}\left[\frac{x_{1}^{\beta_{1}}}{\Omega_{d_{1}}}+\frac{x_{3}^{\beta_{3}}}{\Omega_{d_{3}}}+\frac{(1+\rho) x_{2}^{\beta_{2}}}{\Omega_{d_{2}}}\right]\right\} \\
& \times \sum_{k_{1}, k_{2}=0}^{\infty}\left[\frac{\sqrt{\rho}}{\sqrt[3]{\Omega_{d_{1}} \Omega_{d_{2}} \Omega_{d_{3}}}(1-\rho)}\right]^{2\left(k_{1}+k_{2}\right)} \frac{x_{1}^{\left(k_{1}+1\right) \beta_{1}-1} x_{3}^{\left(k_{2}+1\right) \beta_{3}-1} x_{2}^{\left(k_{1}+k_{2}+1\right) \beta_{2}-1}}{\left(k_{1} ! k_{2} !\right)^{2}}, \\
& f_{y_{1} y_{2} y_{3}}\left(y_{1}, y_{2}, y_{3}\right)=\frac{\beta_{1} \beta_{2} \beta_{3}}{\Omega_{c_{1}} \Omega_{c_{2}} \Omega_{c_{3}}(1-\rho)^{2}} \exp \left\{-\frac{1}{1-\rho}\left[\frac{y_{1}^{\beta_{1}}}{\Omega_{c_{1}}}+\frac{y_{3}^{\beta_{3}}}{\Omega_{c_{3}}}+\frac{(1+\rho) y_{2}^{\beta_{2}}}{\Omega_{c_{2}}}\right]\right\} \\
& \times \sum_{l_{1}, l_{2}=0}^{\infty}\left[\frac{\sqrt{\rho}}{\sqrt[3]{\Omega_{c_{1}} \Omega_{c_{2}} \Omega_{c_{3}}}(1-\rho)}\right]^{2\left(l_{1}+l_{2}\right)} \frac{y_{1}^{\left(l_{1}+1\right) \beta_{1}-1} y_{3}^{\left(l_{2}+1\right) \beta_{3}-1} y_{2}^{\left(l_{1}+l_{2}+1\right) \beta_{2}-1}}{\left(l_{1} ! l_{2} !\right)^{2}},
\end{aligned}
$$

where $\rho$ represents branch correlation coefficient $(0 \leq \rho \leq 1), \beta$ is Weibull fading parameter which expresses fading severity $(\beta>0), \Omega_{d i}=\overline{x_{i}^{\beta_{i}}}$ and $\Omega_{c i}=\overline{y_{i}^{\beta_{i}}}$ are the average powers of desired and interference signal at $i$-th $\operatorname{branch}(i=1,2)$, respectively. $I_{n}(\cdot)$ is the modified Bessel of the first kind and $n$-th order [18, eq. (8.445)].

For the system that chooses the output signal according to DS algorithm, joint PDF of desired signal and interference at the output of dual-branch SC receiver is [12, eq. (4)] 


$$
\begin{aligned}
& f_{x y}(x, y)=y^{\beta_{3}-1} \sum_{i=0}^{\infty} \frac{\beta_{1} \beta_{3}}{\Omega_{d_{1}}^{i+1} \Omega_{c_{1}}} \frac{x^{i \beta_{1}+\beta_{1}-1}}{(i !)^{2}} \exp \left[-\frac{1}{(1-\rho) \Omega_{d_{1}}} x^{\beta_{1}}-\frac{1}{\Omega_{c_{1}}} y^{\beta_{3}}\right] \\
& \times \frac{\rho^{i}}{(1-\rho)^{i}} \gamma\left(i+1, \frac{1}{(1-\rho) \Omega_{d_{2}}} x^{\beta_{2}}\right)+y^{\beta_{4}-1} \sum_{j=0}^{\infty} \frac{\beta_{2} \beta_{4}}{\Omega_{d_{2}}^{j+1} \Omega_{c_{2}}} \frac{x^{j \beta_{2}+\beta_{2}-1}}{(j !)^{2}} \\
& \times \exp \left[-\frac{1}{(1-\rho) \Omega_{d_{2}}} x^{\beta_{2}}-\frac{1}{\Omega_{c_{2}}} y^{\beta_{4}}\right] \frac{\rho^{j}}{(1-\rho)^{j}} \gamma\left(j+1, \frac{1}{(1-\rho) \Omega_{d_{1}}} x^{\beta_{1}}\right) .
\end{aligned}
$$

where $\gamma(a, b)$ represents incomplete Gama function [18, eq. (8.350/1)].

For triple-branch receiver joint PDF of desired signal and interference at the output is [13, eq. (4)]

$$
\begin{aligned}
& f_{x y}(x, y)=A\left[B \sum_{k_{1}, k_{2}=0}^{\infty} x^{\left(k_{1}+1\right) \beta_{1}} \exp \left[-Q_{1} x^{\beta_{1}}\right] \gamma\left(k_{1}+k_{2}+1, Q_{2}(1+\rho) x^{\beta_{2}}\right) \gamma\left(k_{2}+1, Q_{3} x^{\beta_{3}}\right)\right. \\
& +C \sum_{k_{3}, k_{4}=0}^{\infty} x^{\left(k_{3}+k_{4}+1\right) \beta_{2}} \exp \left[-Q_{2}(1+\rho) x^{\beta_{2}}\right] \gamma\left(k_{3}+1, Q_{1} x^{\beta_{1}}\right) \gamma\left(k_{4}+1, Q_{3} x^{\beta_{3}}\right) \\
& \left.+D \sum_{k_{5}, k_{6}=0}^{\infty} x^{\left(k_{6}+1\right) \beta_{3}} \exp \left[-Q_{3} x^{\beta_{3}}\right] \gamma\left(k_{5}+1, Q_{1} x^{\beta_{1}}\right) \gamma\left(k_{5}+k_{6}+1, Q_{2}(1+\rho) x^{\beta_{2}}\right)\right],
\end{aligned}
$$

where

$$
\begin{aligned}
& A=\frac{\beta_{1} \beta_{2} \beta_{3}}{\Omega_{\mathrm{d} 1} \Omega_{\mathrm{d} 2} \Omega_{\mathrm{d} 3}(1-\rho)^{2} x}, Q_{i}=\frac{1}{(1-\rho) \Omega_{\mathrm{di}}}, \\
& C=\frac{\beta_{5}}{\Omega_{c_{2}}} y^{\beta_{5}-1} \exp \left[-\frac{y^{\beta_{5}}}{\Omega_{c_{2}}}\right] \sum_{k_{3}, k_{4}=0}^{\infty} \frac{\left[\frac{\sqrt{\rho}}{\sqrt[3]{\Omega_{\mathrm{d} 1} \Omega_{\mathrm{d} 2} \Omega_{\mathrm{d} 3}}(1-\rho)}\right]^{2\left(k_{3}+k_{4}\right)}}{\left(k_{3} ! k_{4} !\right)^{2} \beta_{1} Q_{1}^{\left(k_{3}+1\right)} \beta_{3} Q_{3}^{\left(k_{4}+1\right)}}, \\
& D=\frac{\beta_{6}}{\Omega_{c_{3}}} y^{\beta_{6}-1} \exp \left[-\frac{y^{\beta_{6}}}{\Omega_{c_{3}}}\right] \sum_{k_{5}, k_{6}=0}^{\infty} \frac{\left[\frac{\sqrt{\rho}}{\sqrt[3]{\Omega_{\mathrm{d} 1} \Omega_{\mathrm{d} 2} \Omega_{\mathrm{d} 3}}(1-\rho)}\right]^{2\left(k_{5}+k_{6}\right)}}{\left(k_{5} ! k_{6} !\right)^{2} \beta_{1} Q_{1}^{\left(k_{5}+1\right)} \beta_{2}\left(Q_{2}(1+\rho)\right)^{\left(k_{5}+k_{6}+1\right)}} .
\end{aligned}
$$

Instantaneous values of SIR on the first, second and third diversity branch are defined as $z_{1}=x_{1} / y_{1}, z_{2}=x_{2} / y_{2}$ and $z_{3}=x_{3} / y_{3}$, respectively. The PDF of instantaneous SIR at the SC output can be calculated using following equation [12, eq (5)]

$$
f_{z}(z)=\int_{0}^{\infty}|J| f_{x y}(z y, y) d y=\int_{0}^{\infty} y f_{x y}(z y, y) d y .
$$

where $|J|$ represents Jacobian of the transformation that can be calculated as 


$$
|J|=\left|\frac{d x}{d z}\right|=y
$$

By substituting (5) in (7) and after integration, final expression for the PDF of instantaneous SIR at the dual-branch receiver is derived as [12, eq. (6)]

$$
\begin{aligned}
& f_{z}(z)=\frac{\beta}{\Omega_{c_{1}}} \sum_{i=0}^{\infty} \frac{\rho^{i}}{(1-\rho)^{i}} \frac{z^{i \beta+\beta-1}}{i ! \Omega_{d_{1}}^{i+1}}\left[\Gamma(i+2)\left(\frac{z^{\beta}}{(1-\rho) \Omega_{d_{1}}}+\frac{1}{\Omega_{c_{1}}}\right)^{-i-2}\right. \\
& \left.-\sum_{m=0}^{i} \frac{1}{m !} \frac{z^{\beta m}}{(1-\rho)^{m}} \frac{\Gamma(i+m+2)}{\Omega_{d_{2}}^{m}}\left(\frac{z^{\beta}}{(1-\rho)}\left(\frac{1}{\Omega_{d_{1}}}+\frac{1}{\Omega_{d_{2}}}\right)+\frac{1}{\Omega_{c_{1}}}\right)^{-i-m-2}\right] \\
& +\frac{\beta}{\Omega_{c_{2}}} \sum_{j=0}^{\infty} \frac{\rho^{j}}{(1-\rho)^{j}} \frac{z^{j \beta+\beta-1}}{j ! \Omega_{d_{2}}^{j+1}}\left[\Gamma(j+2)\left(\frac{z^{\beta}}{(1-\rho) \Omega_{d_{2}}}+\frac{1}{\Omega_{c_{2}}}\right)^{-j-2}\right. \\
& \left.\left.-\sum_{n=0}^{j} \frac{1}{n !} \frac{z^{\beta n}(1-\rho)^{n}}{\Gamma(j+n+2)} \frac{z^{\beta}}{\Omega_{d_{1}}^{n}}\left(\frac{1}{(1-\rho)}+\frac{1}{\Omega_{d_{1}}}\right)+\frac{1}{\Omega_{d_{2}}}\right)^{-j-n-2}\right]
\end{aligned}
$$

where $\Gamma(\alpha)$ represents Gamma function [18, eq. (8.310/1)].

Following the same pattern, by substituting (6) in (7), final expression for the PDF of instantaneous SIR at the triple-branch receiver is [13, eq. (6)]

$$
\begin{aligned}
& f_{z}(z)=\sum_{k_{1}, k_{2}=0}^{\infty} M_{1,2} \frac{z^{\left(k_{1}+1\right) \beta-1}}{\Omega_{c_{1}}} \frac{\left(k_{1}+k_{2}\right) ! k_{2} !}{\left(\frac{W}{\Omega_{\mathrm{d} 2}}\right)^{\left(k_{1}+k_{2}+1\right)}\left(\frac{N}{\Omega_{\mathrm{d} 3}}\right)^{\left(k_{2}+1\right)}}\left[\frac{\Gamma\left(k_{1}+2\right)}{\beta\left(\frac{1}{\Omega_{c_{1}}}+\frac{N}{\Omega_{\mathrm{d} 1}} z^{\beta}\right)^{\left(k_{1}+2\right)}}-\sum_{m=0}^{k_{1}+k_{2}} \frac{\left(\frac{W}{\Omega_{\mathrm{d} 2}} z^{\beta}\right)^{m}}{m ! \beta}\right. \\
& \times \frac{\Gamma\left(k_{1}+m+2\right)}{\left(\frac{1}{\Omega_{c_{1}}}+\frac{N}{\Omega_{\mathrm{d} 1}} z^{\beta}+\frac{W}{\Omega_{\mathrm{d} 2}} z^{\beta}\right)^{k_{1}+m+2}}-\sum_{n=0}^{k_{2}} \frac{\left(\frac{N}{\Omega_{\mathrm{d} 3}} z^{\beta}\right)^{n}}{n ! \beta} \frac{\Gamma\left(k_{1}+n+2\right)}{\left(\frac{1}{\Omega_{c_{1}}}+N z^{\beta}\left(\frac{1}{\Omega_{\mathrm{d} 1}}+\frac{1}{\Omega_{\mathrm{d} 3}}\right)\right)^{k_{1}+n+2}} \\
& \left.+\sum_{m=0}^{k_{1}+k_{2}} \frac{\left(\frac{W}{\Omega_{\mathrm{d} 2}} z^{\beta}\right)^{m}}{m !} \sum_{n=0}^{k_{2}} \frac{\left(\frac{N}{\Omega_{\mathrm{d} 3}} z^{\beta}\right)^{n}}{n !} \frac{\Gamma\left(k_{1}+m+n+2\right)}{\beta\left(\frac{1}{\Omega_{c_{1}}}+\frac{N}{\Omega_{\mathrm{d} 1}} z^{\beta}+N z^{\beta}\left(\frac{(1+\rho)}{\Omega_{\mathrm{d} 2}}+\frac{1}{\Omega_{\mathrm{d} 3}}\right)\right)^{k_{1}+m+n+2}}\right]
\end{aligned}
$$




$$
\begin{aligned}
& +\sum_{k_{3}, k_{4}=0}^{\infty} \frac{z^{\left(k_{3}+k_{4}+1\right) \beta-1}}{\Omega_{c_{2}}} \frac{k_{3} ! k_{4} !}{\left(\frac{N}{\Omega_{\mathrm{d} 1}}\right)^{\left(k_{3}+1\right)}\left(\frac{N}{\Omega_{\mathrm{d} 3}}\right)^{\left(k_{4}+1\right)}}\left[\frac{\Gamma\left(k_{3}+k_{4}+2\right)}{\beta\left(\frac{1}{\Omega_{c_{2}}}+\frac{W}{\Omega_{\mathrm{d} 2}} z^{\beta}\right)^{k_{3}+k_{4}+2}}-\sum_{i=0}^{k_{3}} \frac{\left(\frac{N}{\Omega_{\mathrm{d} 1}} z^{\beta}\right)^{i}}{i !}\right. \\
& \frac{\Gamma\left(k_{3}+k_{4}+i+2\right)}{\beta\left(\frac{1}{\Omega_{c_{2}}}+\frac{W}{\Omega_{\mathrm{d} 2}} z^{\beta}+\frac{N}{\Omega_{\mathrm{d} 1}} z^{\beta}\right)^{k_{3}+k_{4}+i+2}}-\sum_{j=0}^{k_{4}} \frac{\left(\frac{N}{\Omega_{\mathrm{d} 3}} z^{\beta}\right)^{j}}{j !} \frac{\Gamma\left(k_{3}+k_{4}+j+2\right)}{\beta\left(\frac{1}{\Omega_{c_{2}}}+\frac{W}{\Omega_{\mathrm{d} 2}} z^{\beta}+\frac{N}{\Omega_{\mathrm{d} 3}} z^{\beta}\right)^{k_{3}+k_{4}+j+2}} \\
& \left.+\sum_{i=0}^{k_{3}} \frac{\left(\frac{N}{\Omega_{\mathrm{d} 1}} z^{\beta}\right)^{i}}{i !} \sum_{j=0}^{k_{4}} \frac{\left(\frac{N}{\Omega_{\mathrm{d} 3}} z^{\beta}\right)^{j}}{j !} \frac{\Gamma\left(k_{3}+k_{4}+i+j+2\right)}{\beta\left(\frac{1}{\Omega_{c_{2}}}+\frac{W}{\Omega_{\mathrm{d} 2}} z^{\beta}+z^{\beta} N\left(\frac{1}{\Omega_{\mathrm{d} 1}}+\frac{1}{\Omega_{\mathrm{d} 3}}\right)\right)^{k_{3}+k_{4}+i+j+2}}\right] \\
& +\sum_{k_{5}, k_{6}=0}^{\infty} M_{5,6} \frac{z^{\left(k_{6}+1\right) \beta-1}}{\Omega_{c_{3}}} \frac{k_{5} !\left(k_{5}+k_{6}\right) !}{\left(\frac{N}{\Omega_{\mathrm{d} 1}}\right)^{\left(k_{5}+1\right)}\left(\frac{W}{\Omega_{\mathrm{d} 2}}\right)^{\left(k_{5}+k_{6}+1\right)}}\left[\frac{\Gamma\left(k_{6}+2\right)}{\beta\left(\frac{1}{\Omega_{c_{3}}}+\frac{N}{\Omega_{\mathrm{d} 3}} z^{\beta}\right)^{k_{6}+2}}-\sum_{p=0}^{k_{5}} \frac{\left(\frac{N}{\Omega_{\mathrm{d} 1}} z^{\beta}\right)^{p}}{p !}\right. \\
& \frac{\Gamma\left(k_{6}+p+2\right)}{\beta\left(\frac{1}{\Omega_{c_{3}}}+\frac{N}{\Omega_{\mathrm{d} 3}} z^{\beta}+\frac{N}{\Omega_{\mathrm{d} 1}} z^{\beta}\right)^{k_{6}+p+2}}-\sum_{q=0}^{k_{\mathrm{s}+k_{6}}} \frac{\left(\frac{W}{\Omega_{\mathrm{d} 2}} z^{\beta}\right)^{q}}{q !} \frac{\Gamma\left(k_{6}+q+2\right)}{\beta\left(\frac{1}{\Omega_{c_{3}}}+\frac{N}{\Omega_{\mathrm{d} 3}} z^{\beta}+\frac{W}{\Omega_{\mathrm{d} 2}} z^{\beta}\right)^{k_{6}+q+2}} \\
& \left.+\sum_{p=0}^{k_{5}} \frac{\left(\frac{N}{\Omega_{\mathrm{d} 1}} z^{\beta}\right)^{p}}{p !} \sum_{q=0}^{k_{\mathrm{s}}+k_{\mathrm{6}}} \frac{\left(\frac{W}{\Omega_{\mathrm{d} 2}} z^{\beta}\right)^{q}}{q !} \frac{\Gamma\left(k_{6}+p+q+2\right)}{\beta\left(\frac{1}{\Omega_{c_{3}}}+\frac{N}{\Omega_{\mathrm{d} 3}} z^{\beta}+z^{\beta}\left(\frac{N}{\Omega_{\mathrm{d} 1}}+\frac{W}{\Omega_{\mathrm{d} 2}}\right)\right)^{k_{6}+p+q+2}}\right],
\end{aligned}
$$

where

$$
M_{i, j}=\sum_{k_{i}, k_{j}=0}^{\infty} \frac{\beta}{(1-\rho)^{2}}\left[\frac{\sqrt{\rho}}{1-\rho}\right]^{2\left(k_{i}+k_{j}\right)} \frac{1}{\left(k_{i} ! k_{j} !\right)^{2}}, \quad N=\frac{1}{1-\rho}, \quad W=(1+\rho) N .
$$

\section{SYSTEM PERFORMANCE ANALYSIS}

The performance of dual- and triple-branch SC system operating over correlated Weibull fading channels is compared using analytically obtained expressions for the PDF of instantaneous SIR at the output. Outage probability is considered in this paper as performance 
indicator since it is very useful performance measure in wireless communication systems especially when cochannel interference is present. The influence of fading severity, correlation coefficient and average powers is studied and numerical results for outage probability are compared for SC diversity system with two and three branches.

\subsection{Outage probability}

As a basic system performance measure, outage probability, $P_{\text {out }}$, can be defined as the probability that the output SIR drops below a specified threshold $z_{t h}$

$$
P_{\text {out }}=\int_{0}^{z_{\text {th }}} f_{z}(z) d z .
$$

This section analyses the behavior of balanced $\left(S=\Omega_{\mathrm{d} 1} / \Omega_{\mathrm{c} 1}=\Omega_{\mathrm{d} 2} / \Omega_{\mathrm{c} 2}=\Omega_{\mathrm{d} 3} / \Omega_{\mathrm{c} 3}\right)$ dualand triple-branch SC diversity where $S_{\mathrm{i}}=\Omega_{\mathrm{di}} / \Omega_{\mathrm{ci}}$ represents the average SIR at the input of the $i$-th branch of selection combiner.

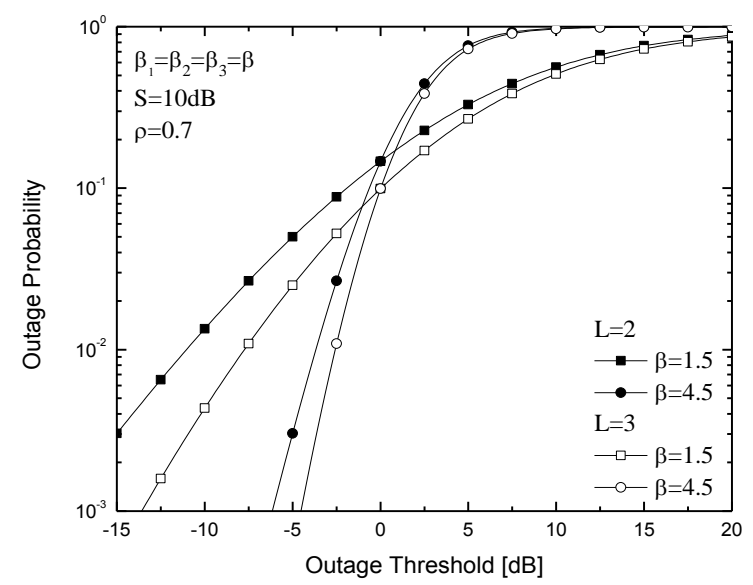

Fig. 1 Influence of fading severity on outage probability for dual- and triple- branch SC system

Fig. 1 represents outage probability of SC receiver with two and three branches that employs desired signal algorithm for different values of fading severity. Outage probability is lower therefore the system performance is better, for higher Weibull fading parameter $\beta$, for the values of outage threshold lower than $0 \mathrm{~dB}$. For higher values of outage threshold, the system performance deteriorates as Weibull fading parameter increases. Also, it can be seen that the outage probability for the system with three branches is lower than the outage probability for the system with two branches.

Relative decrease in outage probability due to larger number of diversity branches can be defined as

$$
\Delta_{n, n+1}=\left[1-\frac{P_{\text {out }_{n+1}}}{P_{\text {out }_{n}}}\right] \cdot 100 \%
$$

For proposed values of $\beta=4.5, S=10 \mathrm{~dB}$ and $\rho=0.7$, relative decrease of outage probability is $\Delta_{2,3}=28.94 \%$ and for $\beta=1.5, S=10 \mathrm{~dB}$ and $\rho=0.7$ there is $\Delta_{2,3}=29.1 \%$ relative decrease. 


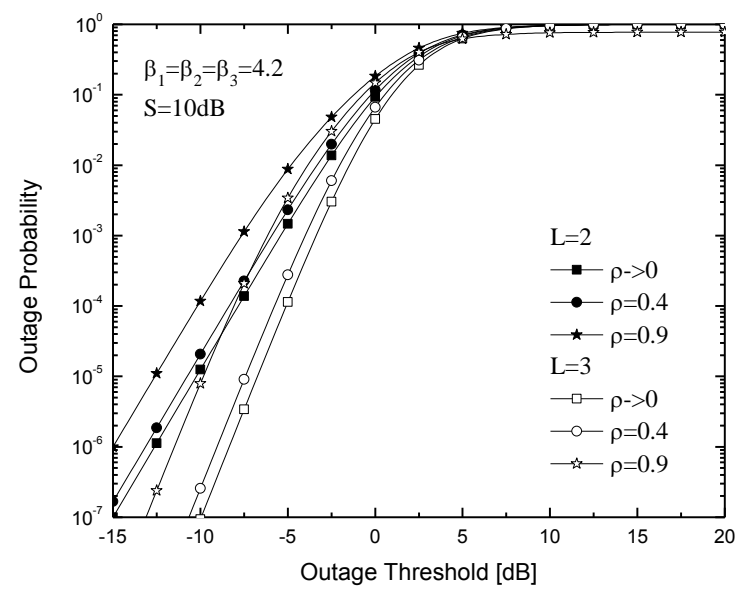

Fig. 2 Influence of correlation coefficient on outage probability for dual- and triple- branch SC system

Fig. 2 illustrates the influence of distance between the diversity branches. The figure confirms that the system performance is better when the distance between the branches is greater. It is noticeable that the system performance is much better when a triple-branch receiver is used. In terms of correlation influence, for the system with no correlation, relative decrease of outage probability is $\Delta_{2,3}=32.67 \%$ while, for the system with high correlation ( $\rho=0.9)$ the relative decrease is $\Delta_{2,3}=21.6 \%$.

The influence of average SIR at the input on outage probability for correlated dual- and triple- branch SC system is presented on Fig. 3. The outage probability decreases as number of branches is higher, which is expected. Also, the increase of average SIR at the input results in better system performance.

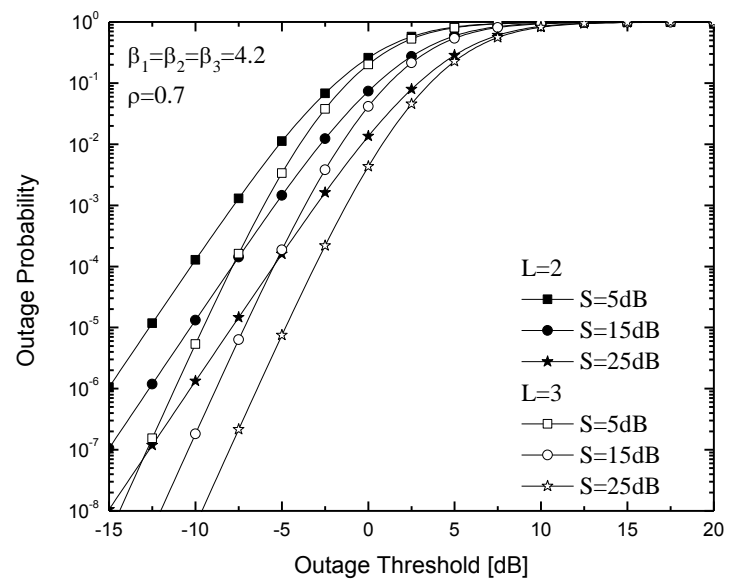

Fig. 3 Influence of average SIR at the input on outage probability for dual- and triple- branch SC system 


\section{CONCLUSION}

This paper compares the outage probability of dual-branch and triple-branch SC receiver operating over correlated Weibull fading channels in the presence of Weibull distributed CCI for the case when desired signal decision algorithm is applied. Numerical results for outage probability were graphically presented describing the influence of correlation coefficient, fading severity and average SIR at the input on overall system performance for both diversity systems. The results obtained for dual-branch and triplebranch receiver were compared showing that system reliability increases as the number of receiving branches changes from two to three.

\section{REFERENCES}

[1] F. Babich, G. Lombardi, "Statistical Analysis and Characterization of the Indoor Propagation Channel", IEEE Trans. Commun., vol. 48, pp. 455-464, Mar. 2000.

[2] H. Hashemi, "The indoor radio propagation channel," Proc. IEEE, vol. 81, pp. 943-968, Jul. 1993.

[3] G. Tzeremes, C. G. Christodoulou, "Use of Weibull Distribution for Describing Outdoor Multipath Fading" Proc. IEEE Anthennas and Propagation society International Symposium 1, pp. 232-235, 2002.

[4] N. S. Adawi, et al., "Coverage prediction for mobile radio systems operating in the $800 / 900 \mathrm{MHz}$ frequency range,” IEEE Trans. Veh. Technol., vol. 37, no. 1, pp. 3-72, Feb. 1988. [Online]. Available:

[5] N. H. Shepherd, "Radio wave loss deviation and shadow loss at $900 \mathrm{MHz}$," IEEE Trans. Veh. Technol., vol. 26, pp. 309-313, Nov. 1977.

[6] M. K. Simon, M. S. Alouini, Digital communications over fading channels, John Wiley \& Sons, Inc. 2000.

[7] Y. Li, X. G. Xia, G. Wang, "Simple iterative methods to exploit the signal-space diversity," IEEE Trans. Commun., vol. 53, no. 1, pp.32-38, Jan. 2005.

[8] J. Boutros, E. Viterbo, "Signal space diversity: A power and bandwidth-efficient diversity technique for Rayleigh fading channel," IEEE Trans. Inf. Theory, vol. 44, pp. 1453-1467, Jul. 1998.

[9] S. H. Lin, T. C. Lee, M. F. Gardina, "Diversity protections for digital radio-summary of ten-year experiments and studies," IEEE Commun. Magazine, vol. 26, no. 2, pp. 51-64, Feb. 1988.

[10] W. Jakes, Microwave mobile communications, John Wiley \& Sons, Inc. 1974.

[11] G. K. Karagiannidis, D. A. Zogas, N. C. Sagias, S. A. Kotsopoulos, G. S. Tombras, "Equal-gain and maximal-ratio combining over nonidentical Weibull fading channels," IEEE Trans. Wireless Commun., vol. 4, no. 3, pp. 841-846, May 2005.

[12] Aleksandra Golubovic, Nikola Sekulovic, Mihajlo Stefanovic, Dejan Milic, Ilija Temelkovski "Performance Analysis of Dual-Branch Selection Diversity Receiver that Uses Desired Signal Algorithm in Correlated Weibull Fading Environment", Tehnicki Vjesnik - Technical Gazette, vol. 21 br. 5, pp. 953-957, 2014.

[13] N. Sekulovic, M. Stefanovic, A. Golubovic, I. Temelkovski, B. Trenkic, M. Peric, S. Milosavljevic „Performance analysis of triple-branch selection diversity based on desired signal algorithm over correlated Weibull fading channels“, TTEM - Technics Technologies Education Management, vol. 7, no. 3, pp. 1013$1019,2012$.

[14] M. H. Ismail and M. M. Matalgah, "Performance of Selection Combining Diversity in Weibull Fading with Cochannel Interference”, EURASIP Journal on Wireless Communications and Networking, pp. 1010, Jan. 2007.

[15] M. C. Stefanovic, D. M. Milovic, A. M. Mitic, M. M. Jakovljevic, "Performance analysis of system with selection combining over correlated Weibull fading channels in the presence of cochannel interference," Int. J. AË̈, vol. 62, no. 9, pp. 695-700, Oct. 2008.

[16] P. Spalevic, N. Sekulovic, Z. Georgios, E. Mekic, "Performance analysis of SIR-based triple selection diversity over correlated Weibull fading Cchannels," Facta Universitatis, Ser.: Elec. Energ. vol. 23, no. 1, pp. 89-98, Apr. 2010.

[17] M. Stefanovic, D. Draca, A. Panajotovic, N. Sekulovic, "Performance analysis of system with L-branch selection combining over correlated Weibull fading channels in the presence of cochannel interference," Int. J. Commun. Systems, vol. 23, no. 2, pp. 139-150, Feb. 2010.

[18] I. Gradshteyn, I. Ryzhik, Table of integrals, series and products, 7Ed, NY: Academic press, 2007.

[19] N. C. Sagias, G. K. Karagiannidis, "Gaussian Class Multivariate Weibull Distributions: Theory and applications in fading channels," IEEE Trans. Inf. Theory, vol. 51, no.10, pp. 3608-3619, Oct. 2005. 